\title{
Augmented Reality in Medical Education and Training
}

\author{
Jennifer Herron, Indiana University School of Medicine, Indianapolis, IN
}

Augmented reality, while not necessarily a new technology, is becoming more well-known and gaining some momentum in medical education through Google Glass and Microsoft's HoloLens. Not only can it aid in student education, it also can impact patient care through its ability to enhance medical training. Medical libraries can partake in this new endeavor by being aware of applications in augmented reality that can benefit students and educators.

Keywords: augmented reality, educational technology, virtual reality

\section{INTRODUCTION}

Many people are somewhat familiar with virtual reality, even more so now with the introduction of head-mounted displays that can give users a virtual reality experience using their smart phones. While virtual reality may be making headlines with these new advancements, its lesser known counterpart, augmented reality, is not as well known. Virtual reality, or VR, is a computer generated environment that fully immerses users and removes their reality. Augmented reality, or AR, utilizes some aspects of virtual reality; however, rather than removing users from their reality, AR allows for virtual presences to be blended into their reality with minimal interference. Microsoft has created the HoloLens, which offers the same immersive experience of headmounted displays, but keeps users' physical reality present. AR applications, or ARAs, that have been presented to students, have been proven to stimulate learners and enhance the learning experience; however, it is not as widely accepted as it seems it should be. AR has great potential

This is the author's manuscript of the article published in final edited form as: Herron, J. (2016). Augmented Reality in Medical Education and Training. Journal of Electronic Resources in Medical Libraries, 13(2), 51-55. http://dx.doi.org/10.1080/15424065.2016.1175987 
in education and especially in medical education given its ability to integrate virtual objects within users' physical reality, making simulations more realistic and immersive.

\section{BACKGROUND}

As futuristic as AR is, it has actually been around for over 50 years. AR first came onto the scene in the 1960's developed by Ivan Sutherland at Harvard University and the University of Utah. ${ }^{1}$ Since this first creation, AR has been developed further in a variety of fields with a numerous applications. AR is a complex system that, in a simple explanation, involves a target activating the virtual trigger which will introduce virtual presences into users' reality. This target can be a patterned marker, a geo-location, object recognition, or voice activated. The most common methods for displaying the virtual aspect of AR are through projection using a see-through display, head-mounted displays, and through handheld devices.

With handheld devices, AR apps are available for smart phones and tablets and typically interact with targets that are printable markers or images, similar to a QR code. The Anatomy 4D app by DAQRI $<$ http://blog.imagine4d.net/project/anatomy-4d/> is one example of an AR app that utilizes an image of a heart with random shapes and markings that when viewed using the app, will trigger a beating heart to appear over the target. Users can interact with the heart by moving the target to manipulate the view of the virtual heart. Moving the targets in AR apps is one method in which users' can interact with the virtual reality aspect of AR. Other examples include modifying surgical equipment with sensors and creating virtual scenarios triggered by targets to test users' ability to correctly utilize the equipment and complete the task involved in the scenario. 


\section{APPLICATIONS IN MEDICAL EDUCATION}

In medical education the phrase, "see one, do one, teach one" is the creed by which students become professionals and go on to help the next generation. ARAs already exist in medical education and subject areas that ARAs cover include anatomy, surgery, and forensic medicine. ${ }^{2}$ Additionally, ARAs have a place in medical training and already assist in actual medical procedures. ARAs have been used to support laparoscopic surgery, endotracheal intubation, joint injections, and assistance in placing local anesthesia. ${ }^{2}$ Numerous studies have been conducted to determine the effective that ARAs have in medical training. One study, conducted at Duke University, developed an educational application of Google Glass to use in conjunction with simulation manikins. ${ }^{3}$ As students made different treatment decisions on the manikins, a video would appear in which an actor portrayed how the patient would respond to the treatment. Students responded very well to the integration of Google Glass into the simulation. One problem that was reported included difficulty in looking at both the real environment and the video that appeared virtually through the Google Glass. Despite this issue, students were very much in favor of the new technology and even had suggestions for additional information to include in the virtual display.

\section{PATIENT CARE AND POTENTIAL APPLICATIONS FOR PATIENT EDUCATION}

Not only does AR have the ability to enhance education and training, but can also lead to better outcomes in patient care. An integrative review completed by Zhu, et al. found that of the over 2,500 papers that they reviewed, $96 \%$ claimed AR to be useful in healthcare education. ${ }^{2}$ Additionally, authors were able to determine that AR increased the speed at which students 
learned and made the learning process easier. When used in training, AR was found to decrease the amount of time needed for practice, provided trainers an outlet for assessment, and increased success rates. ${ }^{2}$

$\mathrm{AR}$ has been discussed in numerous journals regarding its impact on medical training and how it functions in medical and surgical procedures. In one such journal, Orthopedics, a report described the use of AR in a shoulder surgery in which the local surgeon was able to interact with a remote surgeon during the procedure and receive live feedback. Using the Virtual Interactive Presence and Augmented Reality (VIPAAR) system, developed at the University of Alabama at Birmingham, the local surgeon wore Google glass which allowed the remote surgeon to view the procedure and simultaneously allow the local surgeon to see the virtual interaction from the remote surgeon. ${ }^{4}$ The report concluded that the surgery resulted in no complications and illustrates a new and potentially valuable tool in providing additional support for complex procedures and high-risk surgeries. With telemedicine becoming more and more popular, this use of AR shows a promising new development in telemedicine.

AR also has the potential to also be used in patient education. Bifulco, et al. completed a study that tested the use of AR as a teaching tool for people with no medical experience. Researchers created an AR system that enabled people to perform ECG tests by wearing a headmounted display. The AR system used the pattern marker targets to activate virtual placement indicators for the ECG leads. After practicing on a mannequin, participants were then able to perform an actual ECG on a volunteer. Researchers noted that "the average errors in electrode positioning resulted in reasonably acceptable and comparable with placement errors usually made by technicians and nurses in an emergency care department". ${ }^{5}$ 
If training people with no prior medical experience can be made easier and more effective using AR, there are numerous areas in which ARAs could be developed to aid in patient education. Patients requiring regular injections would benefit from an ARA that could provide additional support in training for either themselves or their caregiver. Additionally, ARAs could prove very valuable in instances of medical emergencies by providing quick support for untrained people in which feedback can be provided by remote medical professionals.

\section{LIBRARY ROLE}

AR may seem like something outside the realm of a traditional library, but libraries today are far from traditional. Libraries have gone from checking out books to checking out iPads and even GoPros. Librarians themselves have also changed and transformed their role to interact and engage with their users. Traditional "librarian as teacher" roles have evolved and now include becoming embedded into courses and becoming technology specialists with a need to act as Web site developers, interface designers, and technology integration leaders. ${ }^{6}$ Librarians have taken up these new roles and are creating apps, developing games, and enhancing their websites as a means to provide instruction and information to their users and keep up with educational technology in order to be a relevant resource to students, faculty and instructors.

In these new roles, medical libraries and librarians can provide extra support for faculty and be a 'tech playground' where they can either test out new devices. Libraries can also provide demonstrations of devices or new applications in order to aid instructors in implementing the technology in their classrooms. One of the biggest hurdles to implementing new technology is finding early adopters and instructors willing to create 'products' that demonstrate the value of 
the technology in the learning process. In Guide No. 105, the Association for Medical Education in Europe noted that, "if we remain within the bounds of existing practice, then we cannot expect to gain much from using these technologies" $"$ If libraries are able to provide support to these early adopters by arranging for training sessions or workshops, they can aid in the implementation of new technologies.

Zhu, et al. developed a framework with which to assist in the creation of ARAs in medical education and of the potential needs for applications, two areas stood out in which libraries could become involved. Using AR for role playing interactions with patients was one main area where authors felt an ARA could benefit students. ARAs that allowed both students and practicing professionals to experiment and test theories was another potential ARA found by Zhu et al. ${ }^{7}$ While the library may not become directly involved in the development of these ARAs they can get involved with the acquisition and provide a space for which students and professionals can use them. If libraries can provide the space, similar to study rooms, in which these ARAs can be used they could assist in the adoption of new technology by users and aid them later in their career where this technology may become more abundant.

\section{CONCLUSION}

Medical libraries keep users up to date with the latest medical resources, whether it is e-books, databases, or mobile applications. Expanding their role into technology and helping find relevant uses can be, and in some cases, is, the next area of expertise that medical librarians can provide. By identifying this new technology and making it available for student use and demonstrating its use to faculty can help to revolutionize medical education. It is easy to understand why 
instructors might be weary of implementing a new technology such as AR into their curriculums, but with the support of their libraries it may help build their confidence while also enhancing the connection between librarians, faculty, and students.

\section{REFERENCES}

1. Poelman, R, D.W.F. van Krevelen. "A Survey of Augmented Reality Technologies, Applications and Limitations." The International Journal of Virtual Reality 9, no. 2 (2010): 1-20.

2. Zhu, Egui, Arash Hadadgar, Italo Masiello, and Nabil Zary. "Augmented Reality in Healthcare Education: An Integrative Review." PeerJ 2 (2014): e469-e69.

3. Chaballout, Basil, Margory Molloy, Jacqueline Vaughn, Raymond Brisson III, and Ryan Shaw. "Feasibility of Augmented Reality in Clinical Simulations: Using Google Glass with Manikins." JMIR Medical Education 2, no. 1 (2016): e2.

4. Lindeque, Bennie G. P., Brent A. Ponce, Mariano E. Menendez, Lasun O. Oladeji, Charles T. Fryberger, and Phani K. Dantuluri. "Emerging Technology in Surgical Education: Combining Real-Time Augmented Reality and Wearable Computing Devices." Orthopedics 37, no. 11 (2014): 751-57 7p.

5. Bifulco, Paolo, Fabio Narducci, Raffaele Vertucci, Pasquale Ambruosi, Mario Cesarelli, and Maria Romano. "Telemedicine Supported by Augmented Reality: An Interactive Guide for Untrained People in Performing an Ecg Test." BioMedical Engineering OnLine 13, no. 1 (2014): 1-23.

6. Vassilakaki, Evgenia evasilakaki yahoo gr, and Valentini Moniarou-Papaconstantinou. "A Systematic Literature Review Informing Library and Information Professionals' Emerging Roles." New Library World 116, no. 1/2 (01/ 2015): 37-66.

7. Zhu, Egui, Anneliese Lilienthal, Lauren Aquino Shluzas, Italo Masiello, and Nabil Zary. "Design of Mobile Augmented Reality in Health Care Education: A Theory-Driven Framework." JMIR Medical Education 1, no. 2 (2015): e10.

8. Masters, Ken, Rachel H. Ellaway, David Topps, Douglas Archibald, and Rebecca J. Hogue. "Mobile Technologies in Medical Education: Amee Guide No. 105." Medical Teacher (2016): 1-13. 